\title{
Balance hídrico climático de bosque mesófilo de montaña en la huasteca
}

\section{Climatic water balance of mountain mesophilic forest in the huasteca}

\begin{abstract}
Alberto Santillán-
Fernández ${ }^{1,2 *}$

Samantha Zurisadai Cruz-

Ramírez 2 (D),

Abimael Calva-Castillo2

Arely del Rocío Ireta-

Paredes 3 ,

Jaime Bautista-Ortega 4

${ }^{1}$ Catedrático CONACYT. Cole-

gio de Postgraduados, campus

Campeche. CP. 24450. Cham-

potón, Campeche, México.

${ }^{2}$ División de Ingeniería Forestal.

Instituto Tecnológico Superior de

Venustiano Carranza. CP. 73049.

Venustiano Carranza, Puebla,

México.

${ }^{3}$ Universidad Interserrana del Es-

tado de Puebla-Ahuacatlan.

CP. $73330 . \quad$ San Andrés

Tlayehualancingo, Puebla,

México.

${ }^{4}$ Departamento de Ciencias Agrí-

colas. Colegio de Postgraduados,

campus Campeche. CP. 24450.

Champotón, Campeche, México.

*Autor de correspondencia:

asantillanf@conacyt.mx

Nota científica

Recibido: 12 de octubre de 2018

Aceptado: 06 de febrero de

2020

RESUMEN. Se trabajó en áreas de bosque mesófilo de montaña de los estados de Puebla, Hidalgo y Veracruz, ubicadas dentro de la región Huasteca. Por su naturaleza, el bosque mesófilo de montaña es un buen captador de agua. Pero su cobertura forestal ha disminuido como consecuencia de las actividades antropogénicas, impactando de manera negativa en la captación de agua. Se asoció la evolución temporal (1979-2015) del índice de humedad de las áreas donde existe bosque mesófilo de montaña con los cambios en su cobertura de 1997 a 2016. Los resultados muestran que de 1979 a 2004 el índice de humedad disminuyó como consecuencia de más del 29\% de deforestación. De 2005 a 2016 la tasa de deforestación no superó el $1 \%$ y el índice de humedad presentó una tendencia creciente. Se recomienda la conservación de este ecosistema como prioritario para mejorar la cantidad de agua en la región.

Palabras clave: Déficit, exceso, evapotranspiración, humedad, niebla.

ABSTRACT. Research was conducted in areas of mesophilic mountain forest of Puebla, Hidalgo and Veracruz states, located in the Huasteca region of México. By its nature, the mesophilic mountain forest is a good water collector. But its forest cover decreased as a result of anthropogenic activities, negatively impacting water collection. The temporal evolution (1979-2015) of the humidity index of the areas where there is mesophilic mountain forest was associated with changes in its coverage from 1997 to 2016. The results showed that from 1979 to 2004 the humidity index in the region decreased, as a result of more than $29 \%$ deforestation. From 2005 to 2016 the deforestation rate did not exceed $1 \%$ and the humidity index showed an increasing trend. The conservation of this ecosystem is recommended as a priority to improve the amount of water in the region.
\end{abstract}

Como citar: Santillán-Fernández

A, Cruz-Ramírez SZ, Calva-

Castillo A, Ireta-Paredes AR,

Bautista-Ortega J (2020) Balance

hídrico climático de bosque mesófilo de montaña en la huasteca. Ecosistemas y Recursos Agropecuarios 7(1): e2016. DOI: 10.19136/era.a7n1.2016
Key words: Cloud, deficit, excess, evapotranspiration, moisture. 


\section{INTRODUCCIÓN}

El bosque mesófilo de montaña (BMM), también conocido como bosque de neblina o bosque húmedo de montaña, ocupa menos de $1 \%$ del territorio nacional pero alberga en su cobertura, el $82 \%$ de las familias, $52 \%$ de los géneros y $10 \%$ de las especies de flora vascular de México (Villaseñor 2010). Se desarrolla en regiones con altitudes entre 500 y 2 $800 \mathrm{msnm}$, precipitaciones entre 1000 y $3000 \mathrm{~mm}$ año ${ }^{-1}$, y temperaturas de 12 a $23{ }^{\circ} \mathrm{C}$ (García-De la Cruz et al. 2013). La región Huasteca (límites entre Puebla, Hidalgo y Veracruz) ocupa una extensión que corresponde al $11.78 \%$ del territorio nacional (INEGI 2016).

El BMM es uno de los ecosistemas más importantes por la gran diversidad biológica que posee y los múltiples servicios ecosistémicos que produce como almacenamiento y captura de carbono, regulación de la fertilidad del suelo, control de la erosión, regulación del balance hídrico y escurrimiento hídrico superficial, además del suministro de agua para las ciudades (Álvarez-Zúñiga et al. 2012, Gamfeldt et al. 2013). Por su naturaleza, el principal servicio ecosistémico que proporciona es la captación de agua (Galicia y Zarco-Arista 2013), ya que por la presencia de niebla sobre la vegetación, tiene la propiedad de extraer una cantidad de agua adicional a la que llega en forma de lluvia, de tal manera que, aún en época de estiaje, estos bosques proveen un aporte de agua importante a la hidrología local y regional (CONABIO 2010).

Las evidencias sobre la captura de agua por ecosistemas forestales en Siria (Ungar et al. 2013), Indonesia (Suryatmojo et al. 2013) y España (Del Campo et al. 2014) indican que mejora el balance hídrico. En México Zavaleta et al. (2012) reportan que las áreas forestales regulan el ciclo hidrológico y mejoran la cantidad y calidad de agua que se infiltra a los mantos acuíferos. Pero los BMM, son ecosistemas poco estudiados, por su complejo funcionamiento (Álvarez-Zúñiga et al. 2012). Al respecto, los estudios recientes tratan sobre su dinámica y diversidad (Williams-Linera y VizcaínoBravo 2016, Gual-Díaz y Rendón-Correa 2017), im- portancia de conservación (Álvarez-Aquino et al. 2004, Cayuela et al. 2006, González-Espinosa et al. 2012), efectos de las actividades antropogénicas sobre sus funciones (Williams-Linera et al. 2002, Bautista-Cruz y Del Castillo 2005, Williams-Linera 2007), y resiliencia del ecosistema ante el cambio climático (Foster 2001, Monterroso-Rivas et al. 2013). Pero son pocos los estudios que tratan sobre la dinámica de captación de agua (Manson 2004, Martínez et al. 2009, Muñoz-Villers y McDonnell 2012).

La metodología del Balance Hídrico Climático (BHC), es una herramienta útil para determinar la cantidad de agua que la vegetación dispone, pero también sirve para relacionar la influencia de la vegetación en la captación de agua, al permitir comparar recursos hídricos específicos en un sistema, en diferentes períodos de tiempo (Ruíz-Álvarez et al. 2012). El cálculo de BHC incluye variables de precipitación, temperatura, y propiedades del suelo; y permite conocer la evapotranspiración potencial, exceso y déficit hídrico a lo largo del tiempo (Sentelhas et al. 2008). De acuerdo con Malamos et al. (2015), dentro de los métodos para determinar el $\mathrm{BHC}$, el de más simple cálculo es el propuesto por Thornthwaite y Mather al requerir solo datos de temperatura, precipitación y capacidad de almacenamiento de agua del suelo. Bajo este contexto el objetivo de este estudio fue asociar la evolución temporal del índice de humedad (1979-2015) de las áreas donde existe bosque mesófilo de montaña de la región Huasteca, con los cambios en su cobertura de 1997 a 2016 para determinar la influencia que la cobertura del ecosistema tiene en la captación de agua.

\section{MATERIALES Y MÉTODOS}

\section{Área de estudio}

De la serie VI de uso de suelo y vegetación el Instituto Nacional de Estadística y Geografía (INEGI 2016) se seleccionaron, mediante herramientas de sistemas de información geográfica, las áreas con bosque mesófilo de montaña del norte de Puebla, noroeste de Hidalgo y Norte de Veracruz, ubicadas dentro de la región Huasteca (Figura 1). La región 


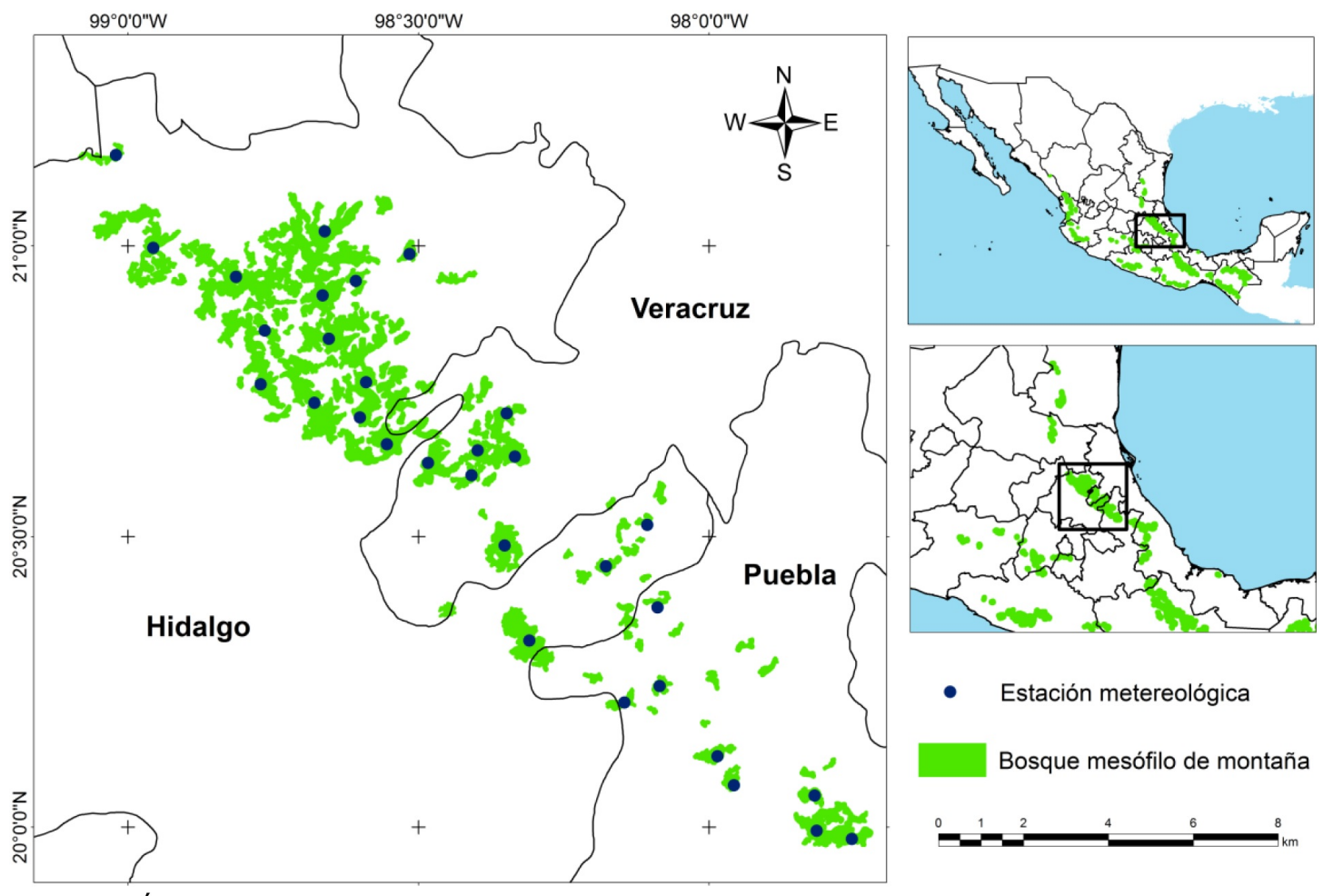

Figura 1. Área de estudio que muestra la distribución geográfica de las estaciones meteorológicas y la cobertura de bosque mesófilo de montaña en la Huasteca.

tiene temperaturas entre los 18 y $25^{\circ} \mathrm{C}$, altitudes superiores a los $1500 \mathrm{msnm}$, con precipitación entre 1000 y $2500 \mathrm{~mm}^{2}$ ño $^{-1}$, y rangos de humedad del 60 al $80 \%$, climas de templados a cálidos en suelos vertisoles; factores que hacen posible el desarrollo de pastizales, selvas y bosques, entre los que se encuentra el bosque mesófilo de montaña. Además de actividades agropecuarias como la ganadería extensiva y el cultivo de naranja, caña de azúcar, tabaco, maíz y frijol (INEGI 2016).

\section{Balance hídrico climático e índice de humedad}

De la base de datos Climatológica Nacional (CLICOM 2018) se seleccionaron las estaciones meteorológicas que estuvieron dentro de las áreas con bosque mesófilo de montaña de la Huasteca y que reportaron información continua de 1979 a 2015. En total se analizaron 31 estaciones meteorológicas: Puebla (8), Hidalgo (17) y Veracruz (6) (Figura 1). De cada estación meteorológica se obtuvo para cada año el promedio mensual de temperatura $\left({ }^{\circ} \mathrm{C}\right)$, precipitación $(\mathrm{mm})$ y evaporación $(\mathrm{mm})$. Esta información sirvió de base para el cálculo regional del Balance Hídrico Climático (BHC) por mes, e índice de humedad por año $(\mathrm{IH})$ con las siguientes metodologías.

Para determinar el BHC se empleó el propuesto por Thornthwaite y Mather, debido a que para su cálculo solo se requieren valores de temperatura, precipitación y Capacidad de Almacenamiento de agua del Suelo (CAS). De acuerdo con Rolim et al. (1998) se promediaron los valores de temperatura y precipitación por mes de las 31 estaciones meteorológicas para la serie 1979-2015 y se empleó un CAS de 200 mm (Santillán et al. 2013), lo que permitió estimar la evapotranspiración potencial (PET), el exceso hídrico (EH) y el déficit hídrico (DH) mensuales de la región Huasteca. Para el cálculo del IH por año expresado en \%, se promediaron por año los valores mensuales de PET, EH y $\mathrm{DH}$, mientras que el IH se calculó con la fórmula propuesta por Ruíz-Álvarez et al. (2012): 


$$
I H=\frac{100(E H-D H)}{P E T}
$$

\section{Índice de humedad y su relación con la cobertura de BMM}

Por su naturaleza los BMM son buenos captadores de agua (CONABIO 2010). Por este motivo, se consideró de manera especial el $\mathrm{IH}$, ya que su cálculo considera la precipitación, temperatura media, evapotranspiración potencial, déficit hídrico y exceso hídrico. Aunque el IH puede verse afectado por eventos aislados como sequias o huracanes, en general expresa la incidencia que la vegetación tiene en la captación de agua (Sentelhas et al. 2008). En virtud de ello, la evolución temporal del IH se asoció con las precipitaciones de 1979 a 2015 y con los cambios en la cobertura del BMM de 1997 a 2016, calculados a partir de las superficies que reportaron las series de uso de suelo y vegetación I, II, III, IV, V y VI del Instituto Nacional de Estadística y Geografía (INEINEGI 1997, INEGI 2001200520092013 у 2016). Los indicadores estadísticos utilizados fueron la Tasa Media de Crecimiento Anual (TMCA), coeficiente de correlación y una prueba de medias por Tukey.

\section{RESULTADOS Y DISCUSIÓN}

\section{Balance hídrico climático}

En la región Huasteca con BMM la precipitación es mayor al potencial de evapotranspiración, lo que provoca que el periodo de estiaje sea mínimo y solo se presente en los meses de febrero a abril, con amplia disponibilidad de agua para la vegetación de mayo a enero (Figura 2). Este hecho contrasta con lo que ocurre en zonas cañeras de la misma región Huasteca, donde de acuerdo con Santillan-Fernández et al. (2016) el déficit hídrico es más prolongado y se presenta de noviembre a junio, por lo que la producción cañera se mantiene gracias al uso de riego. La propiedad de buenos captadores de agua que poseen los BMM ha sido ampliamente documentada por CONABIO (2010), González-Espinosa et al. (2012), Muñoz-Villers et al. (2012), Galicia y Gamfeldt et al. (2013) y Galicia y
Zarco-Arista (2013). Pero ninguno de estos autores relaciona la cobertura de BMM con la capacidad de captación de agua en una región, en lo que sí coinciden es en que la conservación del BMM es importante para mejorar la cantidad y calidad del agua de las regiones aledañas a los BMM.

\section{Índice de humedad y su relación con la cobertura de BMM}

La media del IH de 1979 a 2015 para las zonas de BMM de la región Huasteca fue de $103.51 \%$, de acuerdo a la clasificación de climas propuestos por Thornthwaite corresponde a un Clima A Perhúmedo (Ruíz-Álvarez et al. 2012). Pero como se observa en la Figura 3 , el IH presentó valores menores o iguales al $100 \%$ en 16 de los 37 años analizados, con una TMCA negativa (-2.97\%) de 1979 a 2004 y un valor extremo en 2002 de $-6.53 \%$ que correspondió a un año seco subhúmedo como resultado de la sequía extrema que se presentó ese año en la región (CONAGUA 2018). Estos resultados ayudan a explicar el por qué CONABIO (2017) clasifica a la región de BMM en la Huasteca como una zona de transición entre los climas templado húmedo y semicálido húmedo, altamente vulnerable a los efectos del cambio climático por la inestabilidad en las precipitaciones (Monterroso-Rivas et al. 2013).

Para establecer la relación entre el IH y la cobertura de BMM de la región Huasteca, se dividió la serie 1979-2015 en tres periodos: 1979-1996, 19972004 y 2005-2015, en función de la tendencia del IH y las coberturas de BMM (Figura 4). En cada periodo se asociaron las variaciones del IH (\%), precipitación anual (mm) y cobertura con BMM (ha) (Tabla 1). Los resultados muestran que de 1979 a $2004 \mathrm{el} \mathrm{IH}$, precipitación y cobertura con BMM tuvieron TMCA negativas. El periodo 1997-2004 presentó los valores más bajos para la TMCA del IH con $-5.36 \%$ que coincide con la pérdida en cobertura de BMM de $-29.64 \%$, por explotación principalmente maderable. La correlación entre ambas variables fue de 0.59 , lo que sugiere una relación directa entre el $\mathrm{IH}$ y la cobertura con BMM. Este hecho ha sido documentado por Ungar et al. (2013), Suryatmojo et al. (2013) y Del Campo et al. (2014) quienes encontraron que en ecosistemas 


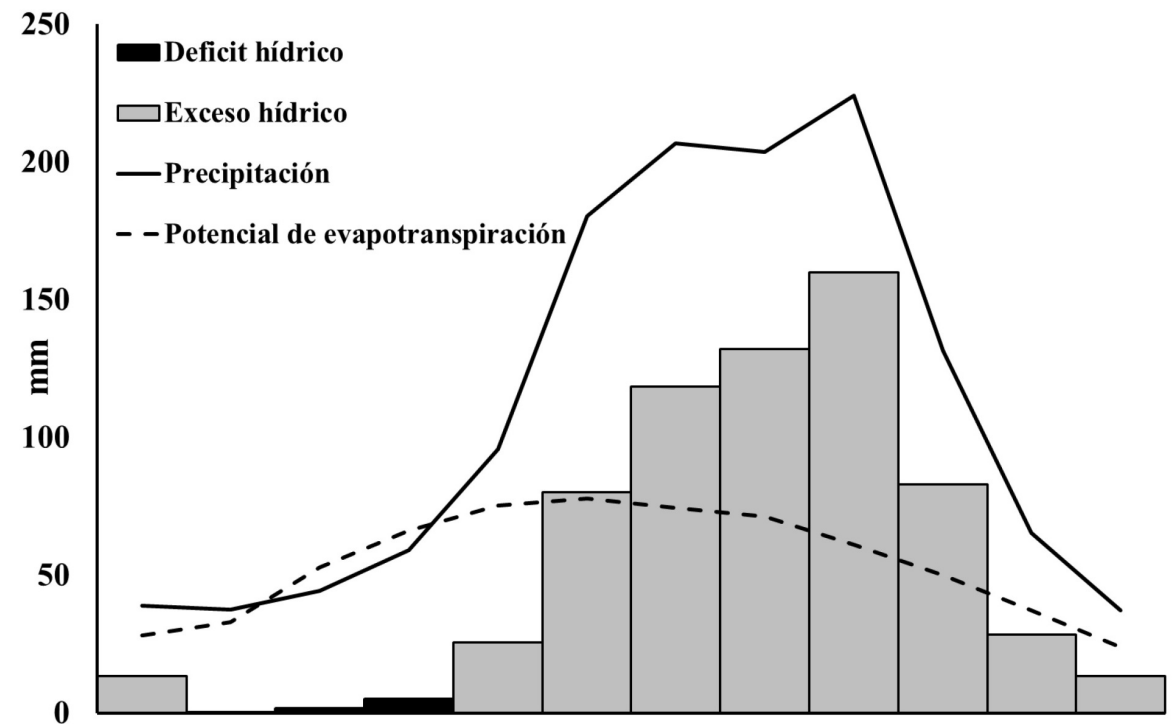

Ene. Feb. Mar. Abr. May. Jun. Jul. Ago. Sep. Oct. Nov. Dic.

Figura 2. Balance hídrico climático de la región Huasteca con bosque mesófilo de montaña construido a partir de los promedios de precipitación, potencial de evapotranspiración, déficit y exceso hídrico de 31 estaciones meteorológicas para el periodo 1979-2015.

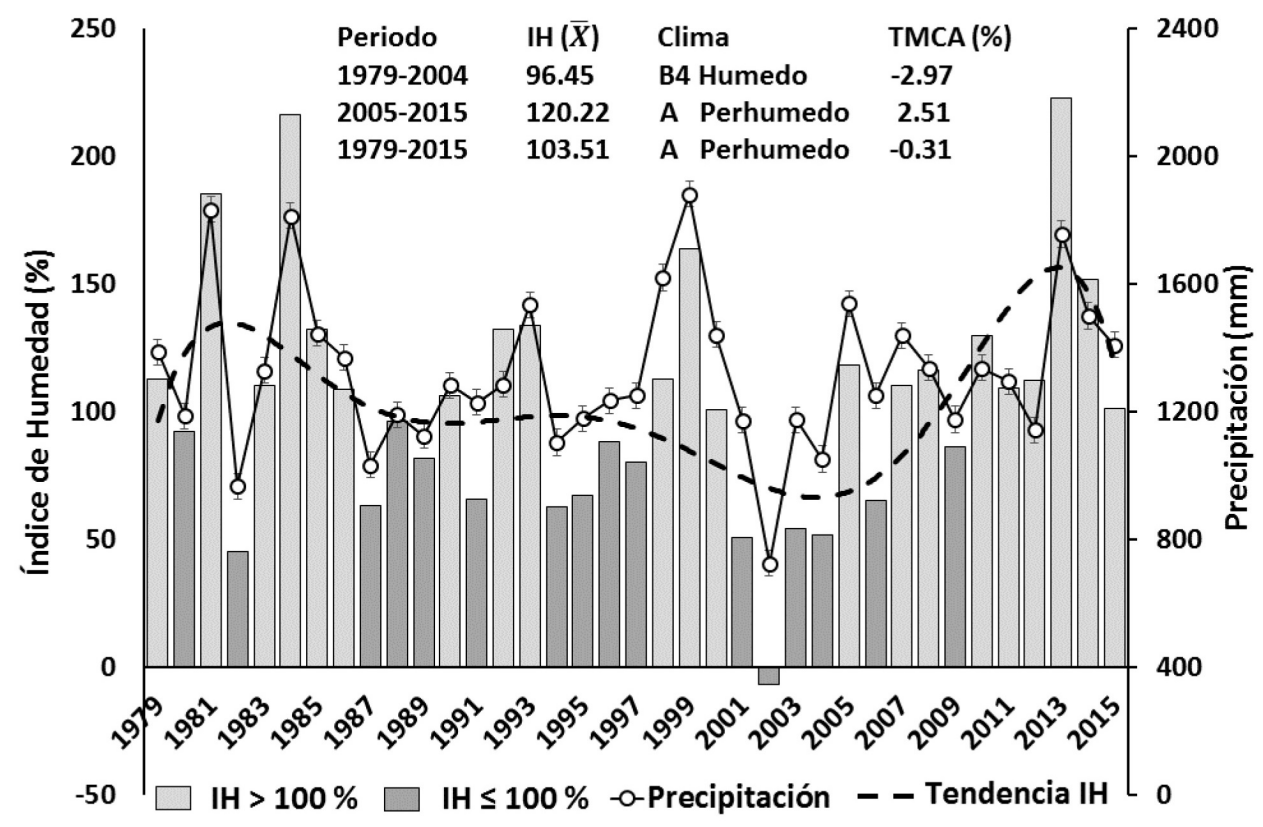

Figura 3. Tendencia del índice de humedad y su relación con la precipitación en la región Huasteca con bosque mesófilo de montaña construido a partir de los promedios de 31 estaciones meteorológicas para el periodo 1979-2015.

forestales a mayor cobertura mayor es el agua captada.

La TMCA positiva (4.49\%) que presentó el IH para el periodo 2005-2015 coincidió con una tasa de deforestación de BMM inferior al 1\% (-0.91\%). De acuerdo con Manson (2004) la captación de agua 


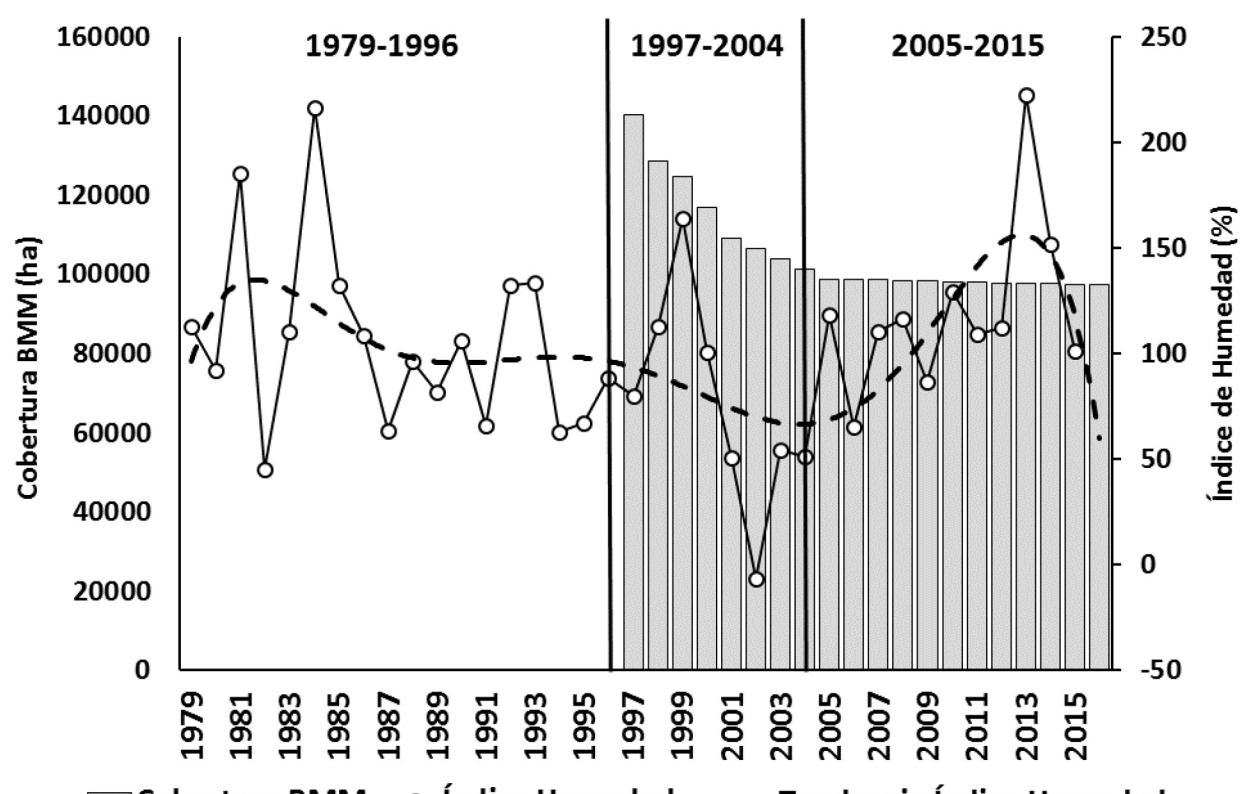

Figura 4. Tendencia del índice de humedad y su relación con la cobertura de bosque mesófilo de montaña en la región Huasteca para los periodos 1979-1996, 1997-2004 y 2005-2015.

Tabla 1. Prueba de medias por Tukey y tasa media de crecimiento anual (\%) para las variables índice de humedad (\%), precipitación ( $\mathrm{mm}$ ) y cobertura de bosque mesófilo de montaña (ha) en la región Huasteca para los periodos 1979-1996, 19972004 y $2005-2015$

\begin{tabular}{|c|c|c|c|c|c|c|c|c|}
\hline \multirow[t]{2}{*}{ Periodo } & \multicolumn{3}{|c|}{$\mathrm{IH}$} & \multicolumn{2}{|c|}{ PRE } & \multicolumn{3}{|c|}{ Cobertura } \\
\hline & Media & TMCA & Clima & Media & TMCA & Media & TMCA & Perdida (\%) \\
\hline 1979-1996 & $105.56 \mathrm{AB}$ & -1.35 & A Perhumedo & $1306.7 \mathrm{~A}$ & -0.64 & & Sin datos & \\
\hline 1997-2004 & $75.94 \mathrm{~B}$ & -5.36 & B3 Humedo & $1289.2 \mathrm{~A}$ & -2.14 & $116372 \mathrm{~A}$ & -3.99 & -29.64 \\
\hline $2005-2015$ & $120.21 \mathrm{~A}$ & 4.49 & A Perhumedo & 1379.6 A & 1.20 & $98135 \mathrm{~B}$ & -0.11 & -0.91 \\
\hline
\end{tabular}

por coberturas forestales es muy sensible a cambios drásticos en sus superficies como el que se presentó de 1997 a 2004 (-29.64\%) y resilente a tasas de deforestación mínimas. Al respecto, Monterroso-Rivas et al. (2013) encontraron que la deforestación de BMM en la Huasteca, a partir de 2002 se debió principalmente al incremento de potreros para la ganadería extensiva, que reconvirtieron zonas de BMM con pastos forrajeros, catalogados como buenos retenedores de agua (Cruz-Martínez et al. 2016). Este hecho aunado a la creación de la Comisión Nacional Forestal en 2001, que dio prioridad a la restauración y conservación del BMM en la Huasteca (CONAFOR 2009), lo que puede explicar que el IH en la región presentara TMCA positivas aunque las lluvias no mostraron incrementos estadísticamente significativos en el pe- riodo de análisis (Tabla 1).

El IH relaciona en su cálculo de manera directa: exceso hídrico, déficit hídrico y potencial de evapotranspiración en una región, y de manera indirecta precipitación y temperatura. Por esta razón se consideró como un indicador de la cantidad de agua que capta el ecosistema de BMM en la región Huasteca. Este hecho permitió determinar la importancia que la cobertura de BMM tiene en el Balance Hídrico Climático regional. Se encontró que el IH tiende a disminuir cuando las coberturas de BMM se reducen agresivamente en periodos cortos, por lo que la restauración y conservación de este ecosistema es prioritario para mejorar la cantidad de agua en la región. Una limitante de la presente investigación es que los cálculos desarrollados provienen 
de información disponible en fuentes oficiales, por lo que se recomienda complementar las metodologías propuestas con datos tomados in situ.

\section{AGRADECIMIENTOS}

A los alumnos de Ingeniería Forestal del Instituto Tecnológico Superior de Venustiano Carranza, Selene Romero Mérida y Velázquez Castillo Yadeli, por su disposición y entusiasmo en el armado de bases de datos y cartografía. Así como a los revisores anónimos que contribuyeron al enriquecimiento del estudio.

\section{LITERATURA CITADA}

Álvarez-Aquino C, Williams-Linera G, Newton AC (2004) Experimental native tree seedling establishment for the restoration of a Mexican cloud forest. Restoration Ecology 12: 412-418.

Álvarez-Zúñiga E, Sánchez-González A, López-Mata L, Tejero-Díez JD (2012) Composición y abundancia de las pteridofitas en el bosque mesófilo de montaña del municipio de Tlanchinol, Hidalgo, México. Botanical Sciences 90: 163-177.

Bautista-Cruz A, Del Castillo RF (2005) Soil changes during secondary succession in a tropical montane cloud forest area. Soil Science Society of America Journal 69: 906-914.

Cayuela L, Golicher DJ, Benayas JMR., González-Espinosa M, Ramírez N (2006) Fragmentation, disturbance and tree diversity conservation in tropical montane forests. Journal of Applied Ecology 43: 1172-1181.

CLICOM (2018) CLImate COMputing Project. Ensenada, B.C. México. http://clicom-mex.cicese.mx/mapa.html. Fecha de consulta 17 de febrero de 2018.

CONABIO (2010) El bosque mesófilo de montaña en México: amenazas y oportunidades para su conservación y manejo sostenible. Comisión Nacional para el Conocimiento y Uso de la Biodiversidad. México. 197p.

CONABIO (2017) Climas. Comisión Nacional para el Conocimiento y Uso de la Biodiversidad, CDMX, México. http://www.conabio.gob.mx/informacion/gis/. Fecha de consulta 28 de enero de 2020.

CONAFOR (2009) Restauración de ecosistemas forestales: guía básica para comunicadores. Comisión Nacional Forestal. Zapopan, Jalisco. México. 69p.

CONAGUA (2018) Monitor de sequía en México. Comisión Nacional del Agua, CDMX, México. https://smn.cna. gob.mx/es/climatologia/monitor-de-sequia/monitor-de-sequia-en-mexico. Fecha de consulta 30 de julio de 2018.

Cruz-Martínez A, Pedroza-Sandoval A, Trejo-Calzada R, Sánchez-Cohen I, Samaniego-Gaxiola JA, HernándezSalgado R (2016) Captación de agua de lluvia y retención de humedad edáfica en el establecimiento de buffel (Cenchrus ciliaris L.). Revista Mexicana de Ciencias Pecuarias 7: 159-172.

Del Campo AD, Fernandes TJ, Molina AJ (2014) Hydrology-oriented (adaptive) silviculture in a semiarid pine plantation: How much can be modified the water cycle through forest management? European Journal of Forest Research 133: 879-894.

Foster P (2001) The potential negative impacts of global climate change on tropical montane cloud forests. EarthScience Reviews 55: 73-106. 
Galicia L, Zarco-Arista AE (2013) Multiple ecosystem services, possible trade-offs and synergies in a temperate forest ecosystem in Mexico: a review. International Journal of Biodiversity Science, Ecosystems Services \& Management 10: 275-288.

Gamfeldt L, Snäll T, Bagchi R, Jonsson M, Gustafsson L, Kjellander P, Ruiz-Jaen MC, Froberg M, Stendahl J, Philipson CD, Mikusinski G, Andersson E, Westerlund B, Andren H, Moberg F, Moen J, Bengtsson J (2013) Higher levels of multiple ecosystem services are found in forests with more tree species. Nature communications 4: 1340. Doi: DOI: 10.1038/ncomms2328.

García-De la Cruz Y, Olivares-López LA, Ramos-Prado JM (2013) Estructura y composición arbórea de un fragmento de bosque mesófilo de montaña en el estado de Veracruz. Revista Chapingo Serie Ciencias Forestales y del Ambiente 19: 91-10.

González-Espinosa M, Meave JA, Ramírez-Marcial N, Toledo-Aceves T, Lorea-Hernández FG, Ibarra-Manríquez G (2012) Los bosques de niebla de México: conservación y restauración de su componente arbóreo. Revista Ecosistemas 21: 36-52.

Gual-Díaz M, Rendón-Correa A (2017) Los bosques mesófilos de montaña de México. Agroproductividad 10: 3-9.

INE-INEGI (1997) Uso del suelo y vegetación, escala 1:250000, serie I. Instituto Nacional de Ecología e Instituto Nacional de Estadística, Geografía e Informática. CDMX, México. http://www.conabio.gob.mx/informacion/ metadata/gis/usv250kcs1agw.xml?_httpcache=yes\&_xsl=/db/metadata/xsl/fgdc_html.xsl\&_indent=no. Fecha de consulta 18 de junio de 2018.

INEGI (2001) Uso del suelo y vegetación, escala 1:250000, serie II. Instituto Nacional de Estadística y Geografía. Aguascalientes, México. http://www.conabio.gob.mx/informacion/metadata/gis/usv250ks2gw.xml?_httpcache=yes\&_xsl=/db/metadata/xsl/fgdc_html.xsl\&_indent=no. Fecha de consulta 18 de junio de 2018.

INEGI (2005) Uso del suelo y vegetación, escala 1:250000, serie III. Instituto Nacional de Estadística y Geografía. Aguascalientes, México. http://www.conabio.gob.mx/informacion/metadata/gis/usv250ks3gw.xml?_httpcache=yes\&_xsl=/db/metadata/xsl/fgdc_html.xsl\&_indent=no. Fecha de consulta 18 de junio de 2018.

INEGI (2009) Uso del suelo y vegetación, escala 1:250000, serie IV. Instituto Nacional de Estadística y Geografía. Aguascalientes, México. http://www.conabio.gob.mx/informacion/metadata/gis/usv250ks4gw.xml?_httpcache=yes\&_xsl=/db/metadata/xsl/fgdc_html.xsl\&_indent=no. Fecha de consulta 18 de junio de 2018.

INEGI (2013) Uso del suelo y vegetación, escala 1:250000, serie V. Instituto Nacional de Estadística y Geografía. Aguascalientes, México. http://www.conabio.gob.mx/informacion/metadata/gis/usv250s5ugw.xml?_httpcache=yes\&_xsl=/db/metadata/xsl/fgdc_html.xsl\&_indent=no. Fecha de consulta 18 de junio de 2018.

INEGI (2016) Uso del suelo y vegetación, escala 1:250000, serie VI. Instituto Nacional de Estadística y Geografía. Aguascalientes, México. http://www.conabio.gob.mx/informacion/metadata/gis/usv250s6gw.xml?_httpcache =yes\&_xsl=/db/metadata/xsl/fgdc_html.xsl\&_indent=no. Fecha de consulta 18 de junio de 2018.

Malamos N, Barouchas PE, Tsirogiannis IL, Liopa-Tsakalidi A, Koromilas T (2015) Estimation of monthly FAO Penman-Monteith evapotranspiration in GIS environment, through a geometry independent algorithm. Agriculture and Agricultural Science Procedia 4: 290-299.

Manson RH (2004) Los servicios hidrológicos y la conservación de los bosques de México. Madera y Bosques 10: 3-20. 
Martínez ML, Pérez-Maqueo O, Vázquez G, Castillo-Campos G, García-Franco J, Mehltreter K et al. (2009) Effects of land use change on biodiversity and ecosystem services in tropical montane cloud forests of Mexico. Forest Ecology and Management 258: 1856-1863.

Monterroso-Rivas A, Gómez-Díaz J, Tinoco-Rueda J (2013) Bosque mesófilo de montaña y escenarios de cambio climático: una evaluación en Hidalgo, México. Revista Chapingo. Serie Ciencias Forestales y del Ambiente 19: 29-43.

Muñoz-Villers LE, McDonnell JJ (2012) Runoff generation in a steep, tropical montane cloud forest catchment on permeable volcanic substrate. Water Resources Research 48: 1-17.

Rolim GS, Sentelhas PC, Barbieri V (1998) Planilhas no ambiente EXCEL TM para os cálculos de balanços hídricos: normal, sequencial, de cultura e de produtividade real e potencial. Revista Brasileira de Agrometeorologia 6: 133-137.

Ruíz-Álvarez O, Arteaga-Ramírez R, Vázquez-Peña MA, Ontiveros-Capurata RE, López-López R (2012) Balance hídrico y clasificación climática del Estado de Tabasco, México. Universidad y Ciencia 28: 1-14.

Santillán GE, Dávila-Vázquez G, De Anda SJ (2013) Estimación del balance hídrico mediante variables climáticas, en la cuenca del río cazones, Veracruz, México. Ambiente y Agua 8: 104-117.

Santillán-Fernández A, Santoyo-Cortés VH, García-Chávez LR, Covarrubias-Gutiérrez I, Merino A (2016) Influence of drought and irrigation on sugarcane yields in different agroecoregions in Mexico. Agricultural Systems 143: 126-135.

Sentelhas PC, Dos Santos DL, Machado RE (2008) Water deficit and water surplus maps for Brazil, based on FAO Penman-Monteith potential evapotranspiration. Ambiente e Agua-An Interdisciplinary Journal of Applied Science 3: 28-42.

Suryatmojo H, Fujimoto M, Yamakawa Y, Kosugi KI, Mizuyama T (2013) Water balance changes in the tropical rainforest with intensive forest management system. International Journal of Sustainable Future for Human Security J-SustaiN 1: 56-62.

Ungar ED, Rotenberg E, Raz-Yaseef N, Cohen S, Yakir D, Schiller G (2013) Transpiration and annual water balance of Aleppo pine in a semiarid region: implications for forest management. Forest ecology and management 298: 39-51.

Villaseñor JL (2010) El bosque húmedo de montaña en México y sus plantas vasculares. Comisión Nacional para el Conocimiento y Uso de la Biodiversidad. CDMX, México. https://www.biodiversidad.gob.mx/publicaciones/ librosDig/pdf/Bosque\%20humedo\%20de\%20montana.pdf. Fecha de consulta 18 de junio de 2018.

Williams-Linera G, Manson RH, Vera El (2002) La fragmentación del bosque mesófilo de montaña y patrones de uso del suelo en la región oeste de Xalapa, Veracruz, México. Madera y Bosques 8: 73-89.

Williams-Linera G (2007) El bosque de niebla del centro de Veracruz: ecología, historia y destino en tiempos de fragmentación y cambio climático. CONABIO - Instituto de Ecología. Xalapa, Veracruz, México. 208p.

Williams-Linera G, Vizcaíno-Bravo Q (2016) Cloud forests on rock outcrop and volcanic soil differ in indicator tree species in Veracruz, Mexico. Revista Mexicana de Biodiversidad 87: 1265-1274.

Zavaleta HE, Cruz-Jiménez H, Márquez-Ramírez J (2012) Potencial de infiltración de agua de lluvia a partir de la retención de una plantación forestal. Foresta Veracruzana 14: 23-28. 